\title{
Prior use of anticoagulation is associated with a better survival in COVID-19
}

\author{
A. G. Buenen ${ }^{1,2}\left(\right.$ Darijn Sinkeldam ${ }^{3} \cdot$ Martje L. Maas $^{4} \cdot$ Martha Verdonschot $^{5} \cdot$ Peter C. Wever $^{6}$
}

Accepted: 18 May 2021 / Published online: 1 June 2021

() The Author(s), under exclusive licence to Springer Science+Business Media, LLC, part of Springer Nature 2021

\begin{abstract}
Coronavirus disease 2019 (COVID-19) is associated with a high incidence of venous and arterial thromboembolic events. The role of anticoagulation (AC) prior to hospital admission and how different types of oral AC influences the outcome of COVID-19 is currently unknown. This observational study compares the outcome in COVID-19 patients with prior use of direct oral anticoagulants (DOAC) or vitamin K antagonists (VKA), and without prior use of AC. We collected the baseline characteristics and outcomes of COVID-19 patients presented to the emergency department of Bernhoven Hospital, the Netherlands. The primary outcome was all-cause mortality within 30 days and analyzed in a multivariable Cox proportional hazards model including age, sex, symptom duration, home medication, and comorbidities. We included 497 patients, including 57 patients with DOAC (11\%) and 53 patients with VKA (11\%). Patients with AC had a lower body temperature and lower C-reactive protein levels. Comparing the primary outcome in patients with AC (DOAC or VKA) and no AC, the adjusted hazard ratio (aHR) was $0.64(95 \% \mathrm{CI} 0.42-0.96, \mathrm{P}=0.03)$. Comparing DOAC and no AC, the aHR was $0.53(95 \% \mathrm{CI}$ $0.32-0.89, \mathrm{P}=0.02$ ) and comparing VKA and no $\mathrm{AC}$, the aHR was $0.77(95 \% \mathrm{CI} 0.47-1.27, \mathrm{P}=0.30$ ). In a subgroup analysis of DOAC, all nine patients with prior use of dabigatran survived within 30 days. In this observational study, the prior use of $\mathrm{AC}$ is associated with a better survival of COVID-19. DOAC, especially dabigatran, might have additional beneficial effects.
\end{abstract}

Keywords COVID-19 $\cdot$ SARS-CoV-2 · Thrombosis · Direct oral anticoagulant · Vitamin K antagonist

\section{Highlights}

- The role of anticoagulation prior to SARS-CoV-2 infection is currently unknown.

A. G. Buenen

Noud.Buenen@mmc.nl

1 Department of Emergency Medicine, Maxima MC, Postbus 7777, 5500 MB Veldhoven, The Netherlands

2 Department of Emergency Medicine, Bernhoven Hospital, Uden, The Netherlands

3 Department of Intensive Care, Canisius-Wilhelmina Hospital, Nijmegen, The Netherlands

4 Department of Internal Medicine, Bernhoven Hospital, Uden, The Netherlands

5 INR Thrombosis Service, Bernhoven Hospital, Uden, The Netherlands

6 Department of Medical Microbiology and Infection Control, Jeroen Bosch Hospital, 's-Hertogenbosch, The Netherlands
- In this observational study the prior use of oral anticoagulation is associated with a better survival of COVID19.

- Direct oral anticoagulants, especially dabigatran, might have additional beneficial effect.

\section{Introduction}

Coronavirus disease 2019 (COVID-19), caused by severe acute respiratory syndrome coronavirus 2 (SARS-CoV-2), results in a high incidence of venous and arterial thromboembolic events [1,2]. The consequences of anticoagulation (AC) on the outcome of COVID-19 has been widely studied and the use of both therapeutic and prophylactic AC is associated with a lower mortality in patients hospitalized with COVID-19 [3, 4]. However, in previous studies the role of AC prior to hospital admission seems not associated with a lower mortality, and it is currently unknown how different types of oral AC influences the outcome of COVID19 [4]. A large nationwide register-based cohort study in 
Sweden showed no beneficial effect from prior direct oral anticoagulant (DOAC) use, but it was unknown if DOAC were continued during the SARS-CoV-2 infection [5]. In a recent large retrospective cohort study from Germany both DOAC and vitamin K antagonists (VKA) were associated with improved clinical outcomes [6]. To further explore the possible benefits of different types of oral $\mathrm{AC}$, we performed this single-center, observational study to assess the survival in COVID-19 patients with prior use of DOAC or VKA compared to patients without prior use of AC.

\section{Methods}

We collected the baseline characteristics and outcome of all COVID-19 patients attending the emergency department (ED) of Bernhoven Hospital, Uden, the Netherlands between March 4 and March 30, 2020. To avoid overcrowded hospitals, Dutch general practitioners (GPs) had an important role in treating patients at home, only severe cases of COVID-19 were referred to hospitals. In addition, several patients were seen at the ED and admitted to dedicated nursing homes with supportive care. Hospital policy was to continue oral AC during hospital admission and corticosteroids were generally not administered.

The diagnosis COVID-19 was made with an in-house real-time reverse transcriptase-polymerase chain reaction targeting the RdRp-gene of SARS-CoV-2 on a deep naso-oropharyngeal swab. Patients had a pharmacy assistant interview in the ED and the home medication was registered in the electronic medical record. Based on the home medication, we primarily categorized patients into two groups: AC or no AC. In a secondary analysis we categorized patients into three groups: DOAC, VKA or no AC. Patients without AC were defined as controls. Also registered was the prior use of statins, renin-angiotensin-aldosterone system (RAAS) inhibitors, and immunosuppressants. The following comorbidities were documented: cardiovascular disease, pulmonary disease, renal disease (GFR <60), hypertension, diabetes mellitus, active malignancy, and obesity (body mass index $\geq 30$ ). Thromboembolic events were documented, but patients were not systematically screened, so thromboembolic events might have been missed.

The primary outcome was defined as all-cause mortality within 30 days after the ED visit and the survival status was ascertained through consultation of a national database. The primary outcome was analyzed in a multivariable Cox proportional hazards model including age, sex, symptom duration, home medication, and comorbidities. We did not include vital parameters and laboratory values in this model, because differences could actually reflect a therapeutic effect of the AC. The types of DOAC were further explored for hypothesis-generating purposes. For statistical analyses, we used IBM SPSS Statistics 26 and results were considered statistically significant at $\mathrm{P}$-value $\leq 0.05$. This study was performed in line with the principles of the Declaration of Helsinki, collected data were held anonymously and the institutional board approved this study. Formal approval from a medical ethics committee was not required, because the medical research involving human subjects act (WMO) does not apply for this observational study.

\section{Results}

During the study period, 497 patients with COVID-19 attended the ED. The overall median age was 72 years, 317 patients $(64 \%)$ were male, and the median symptom duration at ED presentation was 7 days. The prior use of AC was observed in 110 patients (22\%), including DOAC in 57 patients (11\%) and VKA in 53 patients (11\%). Patients with AC were older and had more comorbidities while having a statistically significant shorter duration of symptoms, lower body temperature and lower C-reactive protein (CRP) levels, see Table 1. In the 387 patients without AC, prophylactic low-molecular-weight heparin (LMWH) was started in 193 patients (50\%) and in an additional 31 patients (8\%) therapeutic AC was started in the ED or in hospital. The number of thromboembolic events in patients without $\mathrm{AC}$ and with $\mathrm{AC}$ were comparable.

The overall mortality within 30 days was 31\% (153/497). Comparing the primary outcome in patients with $\mathrm{AC}$ and no $\mathrm{AC}$, the adjusted hazard ratio (aHR) was 0.64 (95\% CI $0.42-0.96, \mathrm{P}=0.03$ ). Comparing DOAC and no AC, the aHR was 0.53 (95\% CI $0.32-0.89, \mathrm{P}=0.02$ ) and comparing VKA and no AC, the aHR was 0.77 (95\% CI 0.47-1.27, $\mathrm{P}=0.30$ ). See Table 2 for other results. The different types of DOAC were further explored. Interestingly, all nine patients with prior use of direct thrombin inhibitor dabigatran survived within 30 days after the ED visit. In a Fisher's exact test the mortality within 30 days in patients with dabigatran $(\mathrm{n}=9)$ was statistically significantly lower $(\mathrm{P}=0.04)$ compared to patients without dabigatran $(n=488)$ despite a higher mean age of 75 years, $67 \%$ male and a high number of comorbidities.

\section{Discussion}

In this observational study, we studied the all-cause mortality within 30 days of COVID-19 patients attending the ED during the first wave of the COVID-19 pandemic in the Netherlands. After adjustment for age, sex, symptom duration, home medication, and comorbidities, the prior use of 
Table 1 Characteristics and outcomes

\begin{tabular}{|c|c|c|c|c|}
\hline & $\begin{array}{l}\text { No AC } \\
(\mathrm{n}=387)\end{array}$ & $\begin{array}{l}\mathrm{AC} \\
(\mathrm{n}=110)\end{array}$ & $\begin{array}{l}\text { DOAC } \\
(n=57)\end{array}$ & $\begin{array}{l}\text { VKA } \\
(\mathrm{n}=53)\end{array}$ \\
\hline \multicolumn{5}{|l|}{ Baseline characteristics } \\
\hline Age in years, median (range) & $70(27-91)$ & $78(44-94) *$ & $77(53-91)^{*}$ & $79(44-94)^{*}$ \\
\hline Male, no. (\%) & $245(63)$ & $72(65)$ & $41(72)$ & $31(58)$ \\
\hline Symptom duration, median (range) & $7(0-22)$ & $7(0-19)^{*}$ & $6(0-19)^{*}$ & $7(0-17)$ \\
\hline \multicolumn{5}{|l|}{ Comorbidities } \\
\hline Cardiovascular disease, no. (\%) & $147(38)$ & $109(99) *$ & $56(98)^{*}$ & $53(100)^{*}$ \\
\hline Pulmonary disease, no. (\%) & $102(26)$ & $27(25)$ & $16(28)$ & $11(21)$ \\
\hline Renal disease, no. (\%) & $62(16)$ & $35(32)^{*}$ & $13(23)$ & $22(42)^{*}$ \\
\hline Hypertension, no. (\%) & $172(44)$ & $87(79)^{*}$ & $40(70)^{*}$ & $47(89)^{*}$ \\
\hline Diabetes mellitus, no. (\%) & $73(19)$ & $29(26)$ & $13(23)$ & $16(30)$ \\
\hline Active malignancy, no. (\%) & $26(7)$ & $9(8)$ & $4(7)$ & $5(9)$ \\
\hline Obesity, no. (\%) & $108(28)$ & $43(39)^{*}$ & $24(42)^{*}$ & $19(36)$ \\
\hline \multicolumn{5}{|l|}{ Home medication } \\
\hline Statin, no. (\%) & $144(37)$ & $65(59)^{*}$ & $31(54)^{*}$ & $34(64) *$ \\
\hline RAAS inhibitor, no. (\%) & $120(31)$ & $59(54)^{*}$ & $28(49)^{*}$ & $31(58)^{*}$ \\
\hline Immunosuppressant, no. (\%) & $27(7)$ & $7(6)$ & $4(7)$ & $3(6)$ \\
\hline \multicolumn{5}{|l|}{ Symptoms } \\
\hline Fever, no. (\%) & $315(81)$ & $84(76)$ & $41(72)$ & $43(81)$ \\
\hline Respiratory complaints, no. (\%) & $346(89)$ & $89(81)^{*}$ & $46(81)$ & $43(81)$ \\
\hline Gastrointestinal complaints, no. (\%) & $211(55)$ & $50(45)$ & $30(53)$ & $20(38)^{*}$ \\
\hline Chest pain, no. (\%) & $75(19)$ & $8(7)^{*}$ & $6(11)$ & $2(4)^{*}$ \\
\hline \multicolumn{5}{|l|}{ Vital signs } \\
\hline NEWS, median & 5 & 5 & 5 & 6 \\
\hline Respiratory rate/min, median & 24 & 24 & 23 & 24 \\
\hline Pulse rate/min, median & 90 & 83 & 87 & 80 \\
\hline Systolic BP, mmHg, median & 136 & 132 & 130 & 136 \\
\hline Body temperature, Celsius, median & 37.8 & $37.4 *$ & $37.3 *$ & 37.5 \\
\hline \multicolumn{5}{|l|}{ Laboratory values } \\
\hline C-reactive protein, $\mathrm{mg} / \mathrm{L}$, median & 91 & $65^{*}$ & $65^{*}$ & 65 \\
\hline Hematocrit, $1 / \mathrm{L}$, median & 0.40 & 0.40 & 0.40 & 0.40 \\
\hline Thrombocytes, $10^{9} / \mathrm{L}$, median & 199 & $187 *$ & 185 & 192 \\
\hline Leukocytes, $10^{9} / \mathrm{L}$, median & 6.5 & 6.6 & 6.6 & 6.6 \\
\hline Neutrophils, $10^{9} / \mathrm{L}$, median & 5.2 & 5.1 & 4.9 & 5.5 \\
\hline Lymphocytes, $10^{9} / \mathrm{L}$, median & 0.9 & 0.9 & 0.9 & $0.7 *$ \\
\hline Ferritin, ug/L, median & 710 & 496 & 461 & 539 \\
\hline Lactate dehydrogenase, U/L, median & 355 & 357 & 299 & 406 \\
\hline Creatine kinase, U/L, median & 133 & 141 & 125 & 142 \\
\hline \multicolumn{5}{|l|}{ Outcome } \\
\hline Hospital admission (\%) & $303(78)$ & $89(81)$ & $43(75)$ & $46(87)$ \\
\hline Death within 30 days (\%) & $107(28)$ & $46(42)^{*}$ & $21(37)$ & $25(47)^{*}$ \\
\hline Thromboembolic event (\%) & $21(5)$ & $6(5)$ & $3(5)$ & $3(6)$ \\
\hline
\end{tabular}

Characteristics of COVID-19 patients categorized into groups

$A C$ anticoagulation, DOAC direct oral anticoagulant, VKA vitamin $\mathrm{K}$ antagonist, RAAS inhibitors reninangiotensin-aldosterone system inhibitors, $N E W S$ national early warning score, $B P$ blood pressure

*Statistically significant different than group 'no AC'
$\mathrm{AC}$ is associated with a better survival (aHR $0.64,95 \% \mathrm{CI}$ $0.42-0.96, \mathrm{P}=0.03$ ). In addition, the prior use DOAC, but not VKA, is associated with a better survival compared to patients without AC. Statin use is also associated with a better survival and the use of immunosuppressants is associated with a worse survival. These results are consistent 
Table 2 Adjusted hazard ratio for home medication in COVID-19 patients

\begin{tabular}{lll}
\hline & $\begin{array}{l}\text { Adjusted hazard ratio } \\
(95 \% \text { CI })\end{array}$ & P-value \\
\hline Primary analysis & & \\
Oral anticoagulant $(\mathrm{n}=110)$ & $0.64(0.42-0.96)$ & 0.03 \\
Statin $(\mathrm{n}=209)$ & $0.63(0.43-0.92)$ & 0.02 \\
RAAS-inhibitors $(\mathrm{n}=179)$ & $1.44(0.95-2.17)$ & 0.09 \\
$\quad$ Immunosuppressant $(\mathrm{n}=34)$ & $2.17(1.17-4.02)$ & 0.01 \\
Secondary analysis & & \\
Oral anticoagulant & & \\
$\quad$ DOAC (n=57) & $0.53(0.32-0.89)$ & 0.02 \\
$\quad$ VKA (n=53) & $0.77(0.47-1.27)$ & 0.30 \\
Statin (n=209) & $0.62(0.42-0.92)$ & 0.02 \\
RAAS-inhibitors $(\mathrm{n}=179)$ & $1.47(0.97-2.24)$ & 0.07 \\
Immunosuppressant $(\mathrm{n}=34)$ & $2.22(1.20-4.11)$ & 0.01 \\
\hline
\end{tabular}

The survival within 30 days of COVID-19 patients was analyzed in a multivariable Cox proportional hazards model including age, sex, symptom duration, home medication, and comorbidities

RAAS-inhibitors renin-angiotensin-aldosterone system inhibitor, $D O A C$ direct oral anticoagulant, $V K A$ vitamin $\mathrm{K}$ antagonist

with previous reports stating that chronic DOAC intake is associated with a decreased mortality in elderly over 70 years of age [7] and over 65 years of age [8]. The mean age of our cohort was 70 years and $81 \%$ of patients were older than 60 years, so these findings may only be relevant for elderly patients. Our results contradict recent registerbased cohort studies from Sweden and Germany [5, 6], but administrative database studies are in general not primarily intended for research purposes and have several limitations [9]. This study adds a new insight that patients with AC had a statistically lower body temperature in the ED and lower CRP levels which might explain the better survival. Limitations in this study are that we mainly assessed severe cases of COVID-19 referred to the ED by GPs, so our observations may not apply to milder cases. In addition, the number of patients and events in this study were small and the observational and single-center design might contribute to unobserved confounding bias.

We can only hypothesize why the prior use of AC seems to be associated with a better survival in COVID-19 patients. The most obvious explanation would be a reduction in overall thromboembolic events. However, we found no differences in thromboembolic events between groups, but differences in microthrombi might still be possible. Alveolar capillary microthrombi were common during autopsy of patients with Covid-19 [10]. Another explanation might be the interaction between inflammation and fibrinolysis. It has been suggested that inhibition of thrombin and coagulation factor Xa prevents a cytokine storm, which has been observed in patients with severe COVID-19 [11]. Patients with $\mathrm{AC}$ had a lower body temperature in the ED and lower CRP levels which could reflect an anti-inflammatory effect. VKA are also known to inhibit thrombin and factor Xa but extrahepatic vitamin $\mathrm{K}$ insufficiency is related to a poor outcome in COVID-19 [12], this might nullify the beneficial effects of VKA and explain the worse survival in our study of patients with VKA compared to DOAC. In a subgroup analysis, all nine patients with direct thrombin inhibitor dabigatran survived within 30 days. Considering that these numbers are very small and need to be interpreted with caution, it is of interest that dabigatran attenuated the development of pulmonary fibrosis in a mouse model [13] and dabigatran mitigated neutrophil extracellular trap (NET)-release which is a key driver in COVID-19 immunothrombosis [14].

This study provides an insight into the association between prior use of oral anticoagulants and the survival of COVID-19 patients. It is currently recommended to administer prophylactic dose LMWH in COVID-19 patients during hospital admission [15]. However, it has shown that hospitalized COVID-19 patients had a high thrombin generation capacity despite heparin therapy [16]. A high thrombin generation was also a hallmark of non-surviving COVID-19 patients with ARDS [17]. It is therefore of interest to explore other forms of AC, the role of DOAC, especially dabigatran, needs attention in further research during different stages of SARS-CoV-2 infection, preferably in prospective randomized controlled trials.

Author contributions AGB designed the study, collected data, interpreted data and wrote the manuscript. MS and PCW collected data and wrote the manuscript. MLM and MV designed the study and wrote the manuscript.

Funding No funding was received.

\section{Declarations}

Conflict of interest The authors report no conflict of interest.

Ethical approval This study was performed in line with the principles of the Declaration of Helsinki, collected data were held anonymously and the institutional board approved this study. Formal approval from a medical ethics committee was not required, because the medical research involving human subjects act (WMO) does not apply for this observational study.

\section{References}

1. Jiménez D, García-Sanchez A, Rali P et al (2021) Incidence of venous thromboembolism and bleeding among hospitalized patients with COVID-19: a systematic review and meta-analysis. Chest 159(3):1182-1196. https://doi.org/10.1016/j.chest.2020.11. 005 
2. Cheruiyot I, Kipkorir V, Ngure B, Misiani M, Munguti J, Ogeng'o J (2021) Arterial thrombosis in coronavirus disease 2019 patients: a rapid systematic review. Ann Vasc Surg 70:273-281. https://doi. org/10.1016/j.avsg.2020.08.087

3. Kamel AM, Sobhy M, Magdy N, Sabry N, Farid S (2020) Anticoagulation outcomes in hospitalized Covid-19 patients: a systematic review and meta-analysis of case-control and cohort studies. Rev Med Virol. https://doi.org/10.1002/rmv.2180

4. Flumignan RLG, Tinôco JD, Pascoal PIF et al (2020) Prophylactic anticoagulants for people hospitalised with COVID-19. Cochr Database Syst Rev 10:CD013739. https://doi.org/10.1002/14651 858.CD013739

5. Flam B, Wintzell V, Ludvigsson JF, Mårtensson J, Pasternak B (2021) Direct oral anticoagulant use and risk of severe COVID-19. J Intern Med 289(3):411-419. https://doi.org/10.1111/joim.13205

6. Fröhlich GM, Jeschke E, Eichler U et al (2021) Impact of oral anticoagulation on clinical outcomes of COVID-19: a nationwide cohort study of hospitalized patients in Germany. Clin Res Cardiol. https://doi.org/10.1007/s00392-020-01783-x

7. Rossi R, Coppi F, Talarico M, Boriani G (2020) Protective role of chronic treatment with direct oral anticoagulants in elderly patients affected by interstitial pneumonia in COVID-19 era. Eur J Intern Med 77:158-160. https://doi.org/10.1016/j.ejim.2020.06. 006

8. Denas G, Gennaro N, Ferroni E et al (2021) Reduction in all-cause mortality in COVID-19 patients on chronic oral anticoagulation: a population-based propensity score matched study. Int J Cardiol 15(329):266-269. https://doi.org/10.1016/j.ijcard.2020.12.024

9. Hashimoto RE, Brodt ED, Skelly AC, Dettori JR (2014) Administrative database studies: goldmine or goose chase? Evid Based Spine Care J 5(2):74-76. https://doi.org/10.1055/s-0034-1390027

10. Ackermann M, Verleden SE, Kuehnel M et al (2020) Pulmonary vascular endothelialitis, thrombosis, and angiogenesis in
Covid-19. N Engl J Med 383(2):120-128. https://doi.org/10.1056/ NEJMoa2015432

11. Jose RJ, Manuel A (2020) COVID-19 cytokine storm: the interplay between inflammation and coagulation. Lancet Respir Med 8(6):e46-e47. https://doi.org/10.1016/S2213-2600(20)30216-2

12. Dofferhoff ASM, Piscaer I, Schurgers LJ et al (2020) Reduced vitamin $\mathrm{K}$ status as a potentially modifiable risk factor of severe COVID-19. Clin Infect Dis. https://doi.org/10.1093/cid/ciaa1258

13. Shea BS, Probst CK, Brazee PL et al (2017) Uncoupling of the profibrotic and hemostatic effects of thrombin in lung fibrosis. JCI Insight 2(9):e86608. https://doi.org/10.1172/jci.insight.86608

14. Skendros P, Mitsios A, Chrysanthopoulou A et al (2020) Complement and tissue factor-enriched neutrophil extracellular traps are key drivers in COVID-19 immunothrombosis. J Clin Invest 130(11):6151-6157. https://doi.org/10.1172/JCI141374

15. Thachil J, Tang N, Gando S et al (2020) ISTH interim guidance on recognition and management of coagulopathy in COVID-19. J Thromb Haemost 18(5):1023-1026

16. Nougier C, Benoit R, Simon M et al (2020) Hypofibrinolytic state and high thrombin generation may play a major role in SARSCOV2 associated thrombosis. J Thromb Haemost 18(9):22152219. https://doi.org/10.1111/jth.15016

17. Ranucci M, Sitzia C, Baryshnikova E et al (2020) Covid-19-associated coagulopathy: biomarkers of thrombin generation and fibrinolysis leading the outcome. J Clin Med 9(11):3487. https:// doi.org/10.3390/jcm9113487

Publisher's Note Springer Nature remains neutral with regard to jurisdictional claims in published maps and institutional affiliations. 